\title{
Outcome of the Use of a Synthetic Mesh in the Repair of Burst Abdomen as Compared with Simple Suture
}

\author{
Manuel López-Cano ${ }^{*}$, José A. Pereira², Xavier Feliu ${ }^{3}$, Lourdes Hernández², \\ Daniel Andrada1, Esther Gil1, Borja Villanueva', Manuel Armengol-Carrasco' \\ ${ }^{1}$ Unidad de Cirugía de la Pared Abdominal, Servicio de Cirugía General y Digestiva, Hospital Universitario Vall \\ d'Hebron, Universitat Autònoma de Barcelona, Barcelona, Spain \\ ${ }^{2}$ Servicio de Cirugía General y Digestiva, Hospital del Mar, Parc de Salut Mar, Departamento de Ciencias \\ Experimentales y de la Salud, Universitat Pompeu Fabra, Barcelona, Spain \\ ${ }^{3}$ Servicio de Cirugía General y Digestiva, Hospital de Igualada, Barcelona, Spain \\ Email: ${ }^{*}$ mlpezcano@gmail.com
}

Received 23 February 2015; accepted 12 March 2015; published 18 March 2015

Copyright (C) 2015 by authors and Scientific Research Publishing Inc.

This work is licensed under the Creative Commons Attribution International License (CC BY).

http://creativecommons.org/licenses/by/4.0/

(c) (i) Open Access

\begin{abstract}
Aim: To assess the outcome (postoperative morbidity and mortality and/or development of incisional hernia) of patients with burst abdomen (BA) of midline laparotomy without intestinal fistula comparing early closure of the abdominal wall with a running suture alone vs. running suture plus reinforcement with a non-absorbable synthetic mesh. Methods: Retrospective analysis of the medical records of all patients undergoing surgical repair of a BA in three hospitals between 2006 and 2011. Risk factors, postoperative complications and the incidence of incisional hernia (IH) after a minimum of 12-month follow-up period were compared between groups. Results: A total of 109 patients were treated for $B A, 18$ patients treated by non-standard procedures were excluded from analysis. Of the remaining 91 patients, 56 belong to the mesh group and 35 to the suture group. The overall rate of postoperative complications was high in both groups $(79.1 \%)$. Mortality rate was higher in the suture group than in the mesh group $(31.4 \%$ vs. $14.3 \%$; $P=0.06)$. IH was also more frequent in the suture group $(36.4 \%$ vs. $17.6 \%$; $P=0.057)$. Conclusions: Patients with $\mathrm{BA}$ and without intestinal fistula could be candidates for surgical repair using a running suture and a non-absorbable polypropylene mesh in the "onlay" position.
\end{abstract}

\section{Keywords}

Mesh, Suture, Burst Abdomen, Abdominal Wall, Hernia

"Corresponding author. 


\section{Introduction}

Burst abdomen (BA) is a well known postoperative complication appearing either immediately or during the first hours or days after a laparotomy [1]. The incidence has been reported between $0.5 \%$ and $3 \%$ of all laparotomies [2] [3]. The mortality is high (34\% - 44\%) [4]-[6] often secondary to early postoperative complications or late complications related to the development of an incisional hernia (IH) in $40 \%-60 \%$ of cases [7] [8]. Two types of BA has been described: 1) complete, in which all layers of the abdominal wall including the skin are involved and 2) incomplete, when the skin remains mostly intact and holds the typical clinical feature of a drainage of serous-bloody secretion [1] [3] [9].

The treatment is based on closing the abdominal wall again [1] [3]. Closing the abdominal wall can be hindered by intestinal edema and/or rigidity of the abdominal wall, intra-abdominal infections and/or the presence of an intestinal fistula [3] [6] [9]-[12]. In this previous context [13], patients can be candidates for 1) delayed closure of the abdominal wall (i.e., "planned incisional hernia"), 2) progressive closure (i.e., dynamic wound closure systems or 3) early closure with or without mesh reinforcement.

Different reviews on this topic can be found in literature [1]-[3] [9] [10]. However, there is a lack of data regarding the usefulness of everlasting materials for early closure of a BA [13] [14].

The aim of this retrospective cohort study was to assess the outcome of patients with BA and without intestinal fistula undergoing early closure of the abdominal wall by means of a running suture or by running suture plus insertion of a non-absorbable synthetic mesh. It was hypothesized that the use of a mesh might be beneficial in terms of postoperative morbidity and mortality and/or development of an IH.

\section{Patients and Methods}

A retrospective cohort observational multicentre study was designed between January 2006 and January 2011. All consecutive patients with BA undergoing early repair were included and divided into two groups: suture group (repair by running suture of the abdominal wall [standard of treatment]) and mesh group (running suture plus placement of a synthetic non-absorbable mesh). The main criterion for the choice of the closure method was the preference of the surgeon. All surgeons who did the closure of the abdominal wall were skilled surgeons. Three University hospitals participated in the study, which was approved by the Ethics Committee of each centre. Data were collected from the electronic medical records. All medical records in which "BA" was codified were retrieved.

Inclusion criteria were patients with BA after a midline laparotomy of both sexes, older than 18 years, without an associated intestinal fistula and with early repair of the abdominal wall. Exclusion criteria were patients without surgical treatment, open abdomen or when a progressive closure was needed.

The abdominal wall of all patients was closed using a running suture of a slow absorbable monofilament material (polydioxanone).

Additionally all patients in the mesh group had a running suture plus a prosthetic mesh inserted in the "onlay" position. A $5 \mathrm{~cm}$ supra-aponeurotic dissection was performed at both edges and the mesh was fixed with continuous polypropylene sutures. Polypropylene meshes adjusted to the size of the laparotomy were always used. Skin closure was possible in all patients and one or two suction drainages were left at the surgeon criteria.

For each patient the following data were recorded: demographics, underlying conditions potentially associated with an increased risk of BA such as chronic obstructive pulmonary disease (COPD), malnutrition (serum albumin level $<3 \mathrm{~g} / \mathrm{dL}$ ), diabetes mellitus, anemia (serum hemoglobin concentration $<13 \mathrm{~g} / \mathrm{dL}$ in men and $<12 \mathrm{~g} / \mathrm{dL}$ in women), active malignancy and sepsis [15], initial surgical procedure (elective or urgent) and type of repair (running suture or running suture associated with insertion of a non-absorbable onlay synthetic mesh). Postoperative complications including mortality within the first 30 postoperative days were evaluated using the Clavien-Dindo classification [16]. Wound infection was defined as the infection of the surgical site that appeared during the first 30 postoperative days. In addition chronic infection was defined as persistence of infection beyond 30 days after surgery. Wound infection (deep or superficial) was defined according to criteria of the Centers for Disease Control [17].

Presence of an IH was compared between groups in patients followed more than 12 months after surgery. The diagnosis of IH was done when IH repair was required or when an IH was observed at follow-up by imaging studies (abdominal ultrasound or computed tomography [CT] scan) and/or clinical examination. 


\section{Statistical Analysis}

The statistical analysis was performed with the statistical package SPSS 20.0 (IBM Inc. Rochester, MN. USA). Quantitative variables are expressed as mean and standard deviation (SD) and the categorical variables as frequencies and percentages. Qualitative variables in the groups of simple suture and mesh repair were compared with the chi-square test $\left(\chi^{2}\right)$ or the Fisher's exact test when needed, and continuous variables with the Student's $t$ test for unpaired data or the Mann-Whitney $U$ test when necessary. The normal distribution of variables was assessed with the Kolmogorov-Smirnov test. Statistical significance was set at $\mathrm{P}<0.05$.

\section{Results}

During the study period 109 patients were eligible and 18 (16.5\%) were excluded for the following reasons: not treated surgically (8 patients), presence of an open abdomen (6 patients) and incorrect codification of the medical records (4 patients) leaving 91 patients for analysis (Figure 1).

There were 56 patients in the mesh group and 35 in the suture group. Baseline characteristics of patients in both groups are shown in Table 1 . The distribution of patients by age and gender was similar. Differences in the type of previous surgery (elective vs. urgent) mean time to diagnosis of BA and underlying risk factors were not observed except for a significant higher percentage of patients with serum albumin levels $<3 \mathrm{~g} / \mathrm{dL}$ in the running suture group $(\mathrm{P}=0.01)$.

The 30 -day mortality rate was higher in the suture group than in the mesh group (31.4\% vs. $14.3 \%$ ) although differences were not statistically significant $(P=0.06)$.

Morbidity was similar between groups. The overall rate of postoperative complications was high (79.1\%) without differences between the two study groups. Complications according to the Clavien-Dindo classification were similar. No cases of re-evisceration were observed (Table 2).

There were not significant differences between groups in the superficial surgical site infection rate (Table 2) and all of them were successfully treated by debridement. Only one patient presented a deep surgical site infection.

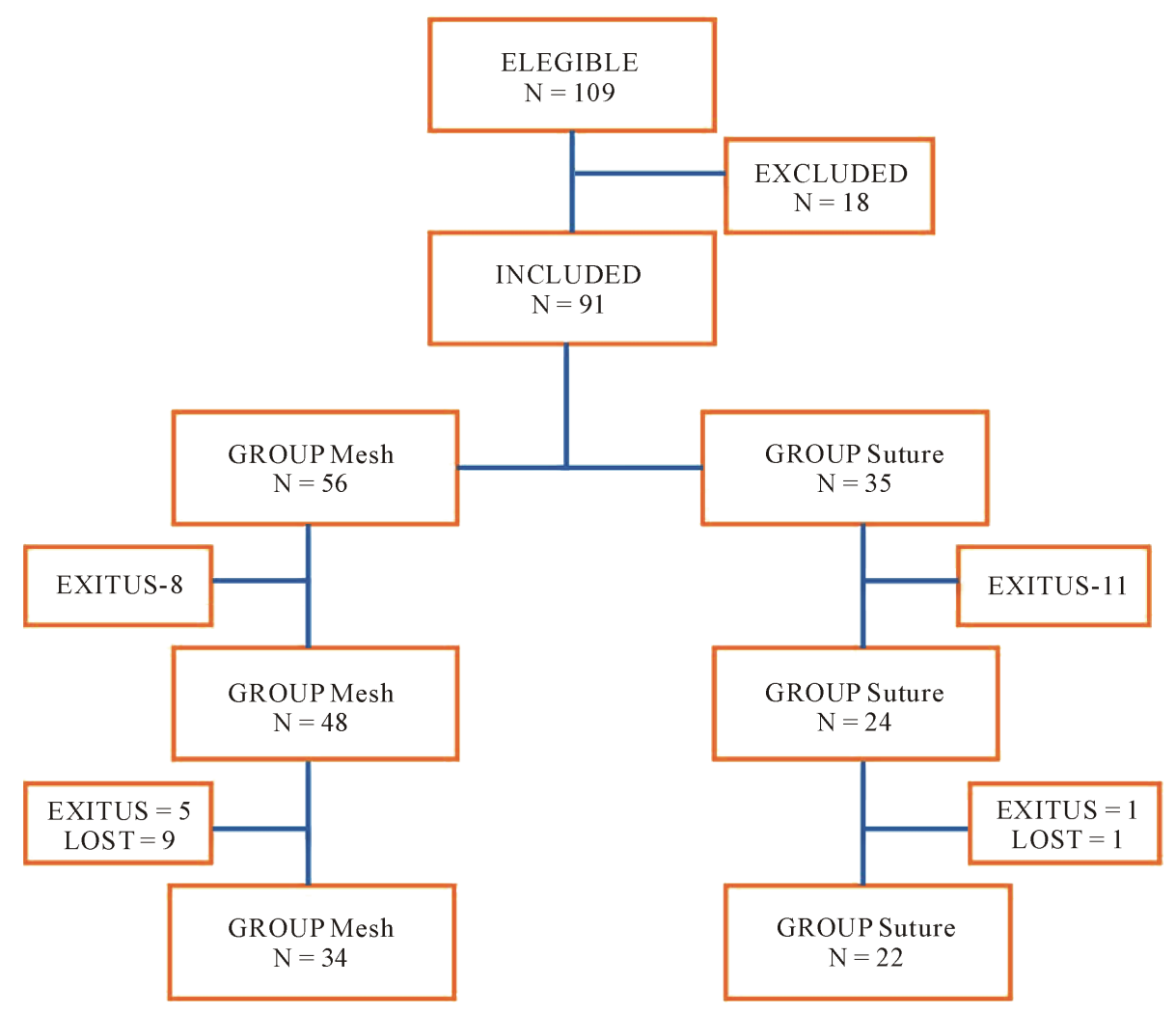

Figure 1. Flow chart of the study participants. 
Table 1. Baseline characteristics of the study groups.

\begin{tabular}{ccccc}
\hline & Total $(\mathrm{N}=91)$ & Mesh $(\mathrm{n}=56)$ & Suture $(\mathrm{n}=35)$ & $\mathrm{P}$ \\
\hline Age: years (SD) & $69.9(12.8)$ & $68.7(14.2)$ & $71.7(10.0)$ & 0.27 \\
Gender: M/F (\%) & $64 / 27(70.3 / 29.7)$ & $38 / 18(67.9 / 32.1)$ & $26 / 9(74.3 / 25.7)$ & 0.34 \\
Initial surgery: emergency/elective (\%) & $34 / 57(37.4 / 62.6)$ & $24 / 32(42.9 / 57.1)$ & $10 / 25(28.6 / 71.4)$ & 0.12 \\
Days to diagnostic: mean (SD) & $9.4(4.8)$ & $9.7(4.9)$ & $8.9(4.6)$ & 0.40 \\
Chronic lung disease: N(\%) & $26(28.6)$ & $15(26.8)$ & $11(31.4)$ & 0.40 \\
Malnutrition (albumin <3 g/dL): N(\%) & $32(35.2)$ & $14(25)$ & $18(51)$ & 0.01 \\
Diabetes: N(\%) & $17(18.7)$ & $9(16.4)$ & $8(22.9)$ & 0.30 \\
Anemia: N(\%) & $51(56)$ & $29(52.7)$ & $22(62.9)$ & 0.23 \\
Neoplasm: N(\%) & $51(56)$ & $33(58.9)$ & $18(51.4)$ & 0.31 \\
Sepsis: N(\%) & $14(15.4)$ & $7(12.5)$ & $7(20)$ & 0.25 \\
\hline
\end{tabular}

Table 2. Comparison of complications.

\begin{tabular}{|c|c|c|c|c|}
\hline & Total $(\mathrm{N}=91)$ & Mesh $(n=56)$ & Suture $(n=35)$ & $\mathrm{P}$ \\
\hline Clavien 0 & 19 (20.9) & $12(21.4)$ & $7(20.0)$ & \multirow{10}{*}{0.15} \\
\hline Clavien I & $2(2.2)$ & $1(1.8)$ & $1(2.9)$ & \\
\hline Clavien II & $20(22)$ & 16 (28.6) & $4(11.4)$ & \\
\hline Clavien III & $28(30.8)$ & $16(28.6)$ & $12(34.3)$ & \\
\hline Clavien IV & $3(3.3)$ & $3(5.4)$ & $0(0)$ & \\
\hline Clavien V & 19 (20.9) & $8(14.3)$ & $11(31.4)$ & \\
\hline Sepsis & 10 & 4 & 6 & \\
\hline MOF $^{*}$ & 3 & 1 & 2 & \\
\hline Respiratory failure & 5 & 2 & 3 & \\
\hline Progression of neoplasia & 1 & 1 & 0 & \\
\hline $\mathrm{SSI}^{* *}$ & $23(25.6)$ & $16(29.1)$ & $7(20.0)$ & 0.45 \\
\hline
\end{tabular}

"MOF: Multiple Organ Failure; ${ }^{* *}$ SSI: Surgical Site Infection.

A total of 56 patients (34 in the mesh group and 22 in the suture group) were followed for more than 12 months postoperatively. IH was developed in 14 (25\%) patients and the percentage between groups was $36.4 \%$ for the suture group vs. $17.6 \%$ for the mesh group $(p=0.057)$. In one patient in whom, a mesh was inserted to treat a BA after a colectomy the mesh had to be explanted due to chronic infection.

\section{Discussion}

BA usually appears in various clinical scenarios precluding an homogeneous treatment [12]. The most common situation is a patient with a postoperative BA appearing during the first 2 weeks after a laparotomy without an associated intestinal fistula [13] [14]. There is no clear evidence regarding the type of treatment that should be systematically used in these cases nor which therapeutic option provides better results. In this scenario, several alternatives have been suggested [13] [14]: Non-surgical treatment and delayed repair of a "planned incisional hernia", closure of the abdominal wall with or without associated retention sutures and/or the insertion of a prosthetic mesh.

The high rate of complications and mortality of BA are well known [3] [8] [14] and have been confirmed in 
our study. A specific complication associated to all types of treatment options is the high rate of IH in the follow-up [14] [18], which is also seen in our series. The use of a synthetic mesh may prevent IH but their use in a setting of contamination or potentially contaminated surgical fields can lead to specific complications such as mesh infection or erosion. However, data from several retrospective studies support the safety of prosthetic mesh insertion in these surgical environments at risk of infection [19]-[21]. In our study, only one mesh required extraction due to chronic infection the rest of patients with surgical site infection were successfully treated without mesh removal.

Our results suggest that the use of a synthetic polypropylene mesh in a "onlay" position for the treatment of BA is safe and provides better results than running suture only in terms of early mortality and development of an $\mathrm{IH}$ at follow-up. Although the high differences in the incidence of IH does not reach statistical significance, they show a positive trend to the group of mesh and probably with a large sample size this trend will be confirmed.

The findings in the current study should be interpreted taking into account several limitations: retrospective design, the small sample size, other non-absorbable synthetic meshes which are not assessed, the malnutrition (as confounding factor) in suture repair group which is significantly higher than in mesh group and the length of follow-up.

The management of BA with abdominal wall closure and mesh reinforcement is an unexplored area within the field of abdominal wall surgery and studies are needed (retrospective series, prospective series, randomized trials) to provide information at all levels of evidence and negate or balance all confounding factors. This retrospective series suggest that in patients with postoperative BA without intestinal fistula the reinforcement of a running suture with a polypropylene mesh in an "onlay" position can be more effective than a running suture alone.

\section{Conflict of Interests}

Authors declare no conflict of interest.

\section{References}

[1] Carlson, M.A. (1997) Acute Wound Failure. Surgical Clinics of North America, 77, 607-636. http://dx.doi.org/10.1016/S0039-6109(05)70571-5

[2] Webster, C., Neumayer, L., Smout, R., Horn, S., Daley, J., Henderson, W. and Khuri, S. (2003) Prognostic Models of Abdominal Wound Dehiscence after Laparotomy. Journal of Surgical Research, 109,130-137. http://dx.doi.org/10.1016/S0022-4804(02)00097-5

[3] Eke, N. and Jebbin, N.J. (2006) Abdominal Wound Dehiscence: A Review. International Surgery, 91, $276-287$.

[4] Tera, H. and Aberg, C. (1975) Relaparotomy. A Ten-Year Series. Acta Chirurgica Scandinavica, 141, 637-644.

[5] Graham, D.J., Stevenson, J.T. and McHenry, C.R. (1998) The Association of Intra-Abdominal Infection and Abdominal Wound Dehiscence. The American Surgery, 64, 660-665.

[6] Keill, R.H., Keitzer, W.F., Nichols, W.K., Henzel, J. and DeWeese, M.S. (1973) Abdominal Wound Dehiscence. Archives of Surgery, 106, 573-577. http://dx.doi.org/10.1001/archsurg.1973.01350160185032

[7] Grace, R.H. and Cox, S. (1976) Incidence of Incisional Hernai after Dehiscence of the Abdominal Wound. The American Journal of Surgery, 131, 210-212. http://dx.doi.org/10.1016/0002-9610(76)90099-4

[8] Van’tRiet, M., De Vos Van Steenwijk, P.J., Bonjer, H.J., Steyerberg, E.W. and Jeekel, J. (2004) Incisional Hernia after Repair of Wound Dehiscence: Incidence and Risk Factors. The American Surgery, 70, 281-286.

[9] Schessel, E.S., Ger, R., Ambrose, G. and King, R. (2002) The Management of the Postoperative Disrupted Abdominal Wall. American Journal of Surgery, 184, 263-268. http://dx.doi.org/10.1016/S0002-9610(02)00935-2

[10] Wahl, W., Menke, H., Schnütgen, M. and Junginger, T. (1992) Fascia Dehiscence-Cause and Prognosis. Der Chirurg, 63, 666-671.

[11] Pavlidis, T.E., Galatianos, I.N., Papaziogas, B.T., Lazaridis, C.N., Atmatzidis, K.S., Makris, J.G. and Papaziogas, T.B. (2001) Complete Dehiscence of the Abdominal Wound and Incriminating Factors. European Journal of Surgery, 167, 351-355. http://dx.doi.org/10.1080/110241501750215221

[12] López-Cano, M., Pereira, J.A. and Armengol-Carrasco, M. (2013) The “Acute Postoperative Open Abdominal Wall”: Nosological Concept and Treatment Implications. World Journal of Gastrointestinal Surgery, 5, 314-320. http://dx.doi.org/10.4240/wjgs.v5.i12.314

[13] vanRamshorst, G.H., Eker, H.H., Harlaar, J.J., Nijens, K.J., Jeekel, J. and Lange, J.F. (2010) Therapeutic Alternatives 
for Burst Abdomen. Surgical Technology International, 19, 111-119.

[14] Abbott, D.E., Dumanian, G.A. and Halverson, A.L. (2007) Management of Laparotomy Wound Dehiscence. The American Surgeon, 73, 1224-1227.

[15] van Ramshorst, G.H., Nieuwenhuizen, J., Hop, W.C.J., Arends, P., Boom, J., Jeekel, J. and Lange, J.F. (2010) Abdominal Wound Dehiscence in Adults: Development and Validation of a Risk Model. World Journal of Surgery, 34, 20-27. http://dx.doi.org/10.1007/s00268-009-0277-y

[16] Dindo, D., Demartines, N. and Clavien, P.A. (2004) Classification of Surgical Complications: A New Proposal with Evaluation in a Cohort of 6336 Patients and Results of a Survey. Annals of Surgery, 240, 205-213. http://dx.doi.org/10.1097/01.sla.0000133083.54934.ae

[17] Mangram, A.J., Horan, T.C., Pearson, M.L., Silver, L.C. and Jarvis, W.R. (1999) Guideline for Prevention of Surgical Site Infection, 1999. Centers for Disease Control and Prevention (CDC) Hospital Infection Control Practices Advisory Committee. American Journal of Infection Control, 27, 97-132. Quiz 133-134; Discussion 196.

[18] Gislason, H. and Viste, A. (1999) Closure of Burst Abdomen after Major Gastrointestinal Operations-Comparison of Different Surgical Techniques and Later Development of Incisional Hernia. European Journal of Surgery, 165, 958961. http://dx.doi.org/10.1080/110241599750008071

[19] Cobb, W.S., Carbonell, A.M., Kalbaugh, C.L., Jones, Y. and Lokey, J.S. (2009) Infection Risk of Open Placement of Intraperitoneal Composite Mesh. The American Surgeon, 75, 762-768.

[20] Carbonell, A.M. and Cobb, W.S. (2013) Safety of Prosthetic Mesh Hernia Repair in Contaminated Fields. Surgical Clinics of North America, 93, 1227-1239. http://dx.doi.org/10.1016/j.suc.2013.06.012

[21] Argudo, N., Pereira, J.A., Sancho, J.J., Membrilla, E., Pons, M.J. and Grande, L. (2014) Prophylactic Synthetic Mesh Can Be Safely Used to Close Emergency Laparotomies, Even in Peritonitis. Surgery, 156, 1238-1244.

http://dx.doi.org/10.1016/j.surg.2014.04.035 